\title{
Applying Neural Network in Classifying Parkinson's Disease
}

\author{
Yuan-Liang (Tom) Liao \\ Fenglinlvzhou F01-10D, Beijing, China \\ Email: yuanliangliao@qq.com
}

How to cite this paper: Liao, Y.-L. (2020) Applying Neural Network in Classifying Parkinson's Disease. Journal of Computer and Communications, 8, 19-23.

https://doi.org/10.4236/jcc.2020.810003

Received: August 22, 2020

Accepted: October 24, 2020

Published: October 27, 2020

Copyright $\odot 2020$ by author(s) and Scientific Research Publishing Inc. This work is licensed under the Creative Commons Attribution International License (CC BY 4.0).

http://creativecommons.org/licenses/by/4.0/

\begin{abstract}
This project uses knowledge of neural network to analyze if the person under study is analyzed to be Parkinson disease patient or not. Binary classification is constructed based on the multi-feature database. A decision boundary is clearly plotted to separate patient with and without Parkinson disease. Results show that over $80 \%$ accuracy could be obtained with the preliminary results. Future efforts could be performed to construct more complicated neural network to improve the accuracy.
\end{abstract}

\section{Keywords}

Machine Learning, Linear Algebra, Error Analysis, Neural Network

\section{Introduction}

Neural network or artificial neural network is the term of network or mathematical model for information functions similar to human brains [1] [2]. In computer science, it is like an artificial human nervous system for receiving, transmitting, and processing information [3] [4]. With the advent of computer science, specifically machine learning, methods have been established to build human brain models based on real world examples to make analysis or decisions. These methods do not require explicit theory but accurate models and training examples. Using different mathematical algorithms and appropriate models, human can make analysis on many things, even identifying cancer, diabetes [2] [4] [5]. Within the realm of machine learning, neural network in a whole is a complex matrix and is set up in layers. Columns are representing each layer and rows determine the length of this matrix. Layers are form of many nodes which are interconnected and contain an activation function. The patterns are presented to the network through the input layer. Like component normal matrix, neutral network function by manipulation of the first column vectors to 
the next column vector. Output would be the last column vector. The computer would use the program between neuron and nodes. The whole process is method of machine learning.

On the other hand, Parkinson's disease is a long-term disease that cause disorder of nervous system which eventually results in shaking, rigidity, and slowness of moving. As the disease worsen, the symptom would cause thinking and behavioral problems. It would be too late to prevent Parkinson's disease when people have already experienced behavioral problems. In early symptoms of Parkinson's disease, it is difficult identify Parkinson since shaking and rigidity can also be regarded to other diseases. Therefore, it is significant to identify Parkinson's disease in order to prevent it. There have been some researches going on the prediction of Parkinson's disease, using multistate Markov models [6], neuropsychological method [7], particle swarm optimization [8], etc. In this paper, we employed artificial neural network and performed a preliminary analysis on the Parkinson's disease. Results show that $83 \%$ accuracy is achieved. In the future, more complicated neural network could be used to further increase the accuracy.

\section{Methods}

We employed sixteen features as the basis for predicting Parkinson's disease. We set each of the features as a variable and make our data of each patients intentionally consist of sixteen numbers. Those sixteen numbers are regarded as the information of patient and we will use it to determine if the person has Parkinson's disease.

Here, we did not simply use matrix and plugging in the data for calculation, a method researcher usually use when they need data analysis. This is because the matrix would be too complicated if we create a matrix with sixteen columns and sample number of rows. Instead, we use another attempt by sixteen features of the patients by creating a $4 \times 4$ square graph shown in Figure 1 . The graph consists of sixteen cells and each of them correspond to a feature. This part is for loading and visualizing the data, making our calculation look less complicate and easy to manipulate.

$$
a_{i}^{(j)}=\text { activation of unit } i \text { in layer } j
$$

$\theta_{j}=$ matrix of weight controlling function mapping from layer $j$ to layer $j+1$

$$
\begin{gathered}
a_{0}^{(2)}=0, \text { biased unit } \\
a_{1}^{(2)}=g\left(\theta_{10}^{(1)} x_{0}+\theta_{11}^{(1)} x_{1}+\theta_{12}^{(1)} x_{2}+\cdots+\theta_{1 n}^{(1)} x_{n}\right) \\
a_{2}^{(2)}=g\left(\theta_{20}^{(1)} x_{0}+\theta_{21}^{(1)} x_{1}+\theta_{22}^{(1)} x_{2}+\cdots+\theta_{2 n}^{(1)} x_{n}\right) \\
a_{n}^{(2)}=g\left(\theta_{n 0}^{(1)} x_{0}+\theta_{n 1}^{(1)} x_{1}+\theta_{2}^{(1)} x_{2}+\cdots+\theta_{n n}^{(1)} x_{n}\right) \\
h_{\theta}(x)=\frac{1}{1-\exp (-x \theta)}=g(x \theta)=g(z)=\frac{1}{1-\exp (-z)}
\end{gathered}
$$




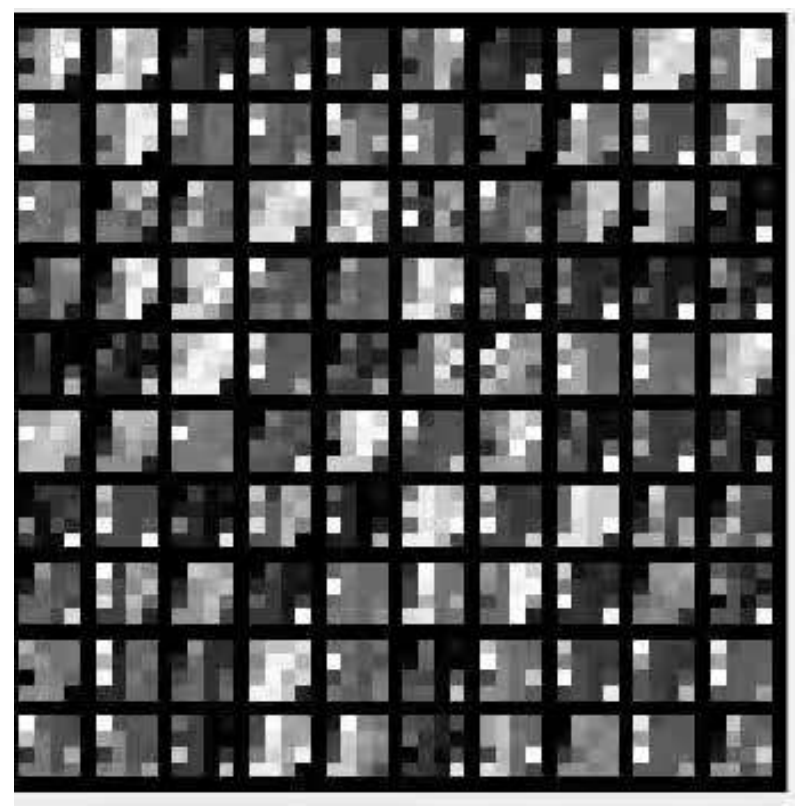

Figure 1. Inputting feature data into $4 \times 4$ matrix graph.

Neural network is a multi-lane approach to model the result of specific relationship [9] [10]. Simply, we have a set of data. Each dependent value corresponds to many another independent variable. From one dependent value we may go to different independent variable and from that independent variable, we may even stretch to more independent variables. Our purpose is to use equation to connect the dependent variable and independent variables, which ultimately provides the result. In this case, we need the features to determine if one have Parkinson's disease. This is not simple programming because the method is temporary and too simple. We are going to tell the computer how to determine, which is regarded as machine learning. Nevertheless, unlike any functions we familiar the most, the variable does not have a direct relationship such as linear or exponential.

We can take the dependent variables connecting to different variable as layers. Neural network contains many layers and thus we have equation of the relationship of these layers. This is how people use the first independent variable to reach the dependent variable, which is also called neuron.

We employed a binary classification method to classify the patients with and without Parkinson's disease. In the first stage, we trained the model by using $90 \%$ of the whole database. We then use the remaining $10 \%$ database as the test data.

\section{Results}

The analysis of the Parkinson's disease with sample data of patient is shown in Figure 1. The shades and spot on the graph show the features of we inputted, also proving that we correctly input the feature of different patient, since the cells have various darkness. We have error analysis as usual by comparing the 
predicted value to real value and gain the accuracy of $82 \%$. It might happen because we only have preliminary data and sixteen features-Parkinson's disease may require more features to analyze. Figure 2 show the scattered data points for patients with and without Parkinson disease. They are represented by the black crosses and yellow solid dots, respectively.

An artificial neural network is then established for training the model and getting the parameters for building the network. The decision boundary can then be determined based, shown in Figure 3 for details. With all that, we calculated the rest of the 10\% database for testing our results. The accuracy for the test results is same as before.

\section{Conclusion}

In this paper, we employed neural network methods combined with feature normalization to accurately determine the patient who has Parkinson disease. Slightly less than $20 \%$ prediction error is acceptable, although. This is a preliminary study,

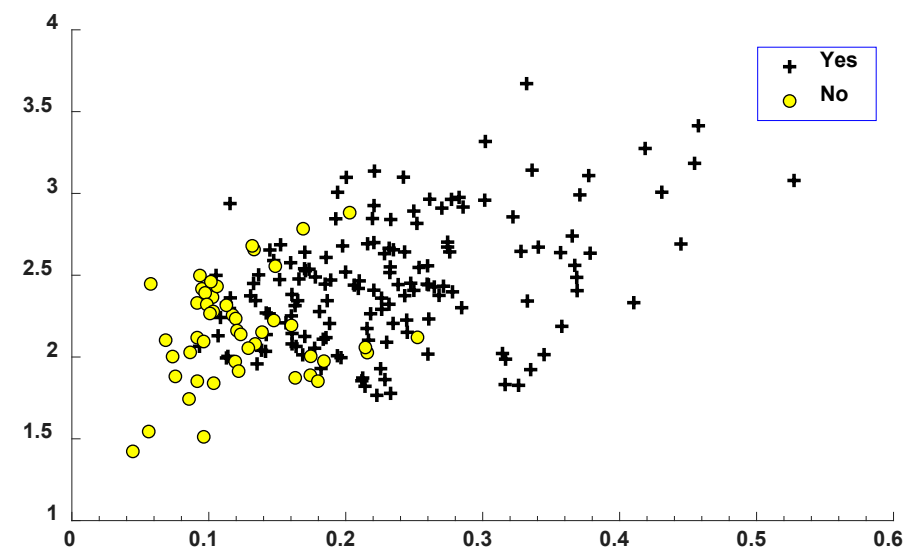

Figure 2. Scattered points for patients with (Black cross) and without (Yellow dots) Parkinson disease.

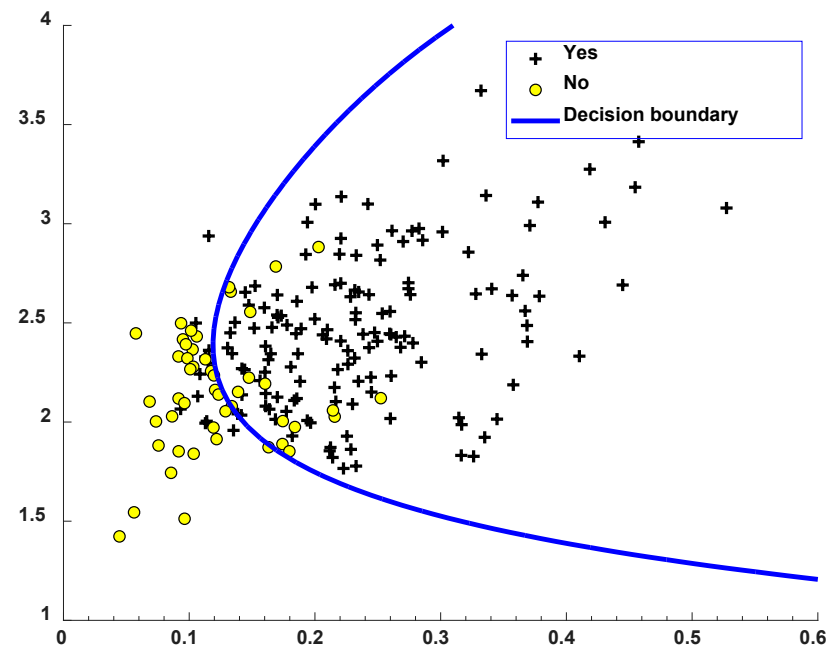

Figure 3. Decision boundary for determine Parkinson disease. 
with more complicated neural network method by incorporating more unknown variable and circumstances, the result will be more difficult to analyze. This method is an imitation of a useful application that can be implemented in our daily life. In addition, the second method seems abstract but it provides a general idea to our real life.

\section{Conflicts of Interest}

The author declares no conflicts of interest regarding the publication of this paper.

\section{References}

[1] Hill, T., Marquez, L., O’Connor, M. and Remus, W. (1994) Artificial Neural Network Models for Forecasting and Decision Making. International Journal of Forecasting, 10, 5-15. https://doi.org/10.1016/0169-2070(94)90045-0

[2] El-Jerjawi, N.S. and Abu-Naser, S.S. (2018) Diabetes Prediction Using Artificial Neural Network. Journal of Advanced Science, 124, 1-10.

[3] Al-Mubayyed, O.M., Abu-Nasser, B.S. and Abu-Naser, S.S. (2019) Predicting Overall Car Performance Using Artificial Neural Network. International Journal of Academic and Applied Research (IJAAR), 3, 1-5.

[4] Nasser, I.M. and Abu-Naser, S.S. (2019) Lung Cancer Detection Using Artificial Neural Network. International Journal of Engineering and Information Systems (IJEAIS), 3, 17-23.

[5] Kashf, D.W.A., Okasha, A.N., Sahyoun, N.A., El-Rabi, R.E. and Abu-Naser, S.S. (2018) Predicting DNA Lung Cancer Using Artificial Neural Network. International Journal for Academic Development, 2, 6-13.

[6] Costin, H. and Geman, O. (2013) Parkinson's Disease Prediction Based on Multistate Markov Models. International Journal of Computers Communications \& Control, 8, 525-537. https://doi.org/10.15837/ijccc.2013.4.498

[7] Mahieux, F., Fénelon, G., Flahault, A., Manifacier, M.-J., Michelet, D. and Boller, F. (1998) Neuropsychological Prediction of Dementia in Parkinson's Disease. Journal of Neurology, Neurosurgery \& Psychiatry, 64, 178-183.

https://doi.org/10.1136/jnnp.64.2.178

[8] Wu, D.F., Warwick, K., Ma, Z., Gasson, M.N., Burgess, J.G., Pan, S. and Aziz, T.Z. (2010) Prediction of Parkinson's Disease Tremor Onset Using a Radial Basis Function Neural Network Based on Particle Swarm Optimization. International Journal of Neural Systems, 20, 109-116. https://doi.org/10.1142/S0129065710002292

[9] Maksimenko, V.A., Kurkin, S.A., Pitsik, E.N., Musatov, V.Yu., Runnova, A.E., Efremova, T.Yu., Hramov, A.E. and Pisarchik, A.N. (2018) Artificial Neural Network Classification of Motor-Related EEG: An Increase in Classification Accuracy by Reducing Signal Complexity. Complexity, 2018. https://doi.org/10.1155/2018/9385947

[10] Alghoul, A., Al Ajrami, S., Al Jarousha, G., Harb, G. and Abu-Naser, S.S. (2018) Email Classification Using Artificial Neural Network. International Journal for Academic Development, 2, 8-14. 